\title{
RISK FACTORS OF REPEAT SHUNT-RELATED CEREBROSPINAL FLUID INFECTIONS IN CHILDREN
}

\author{
A. Yilmaz ${ }^{1}$, A.M. Musluman ${ }^{1}$, N. Dalgic ${ }^{2}$, T. Cansever ${ }^{3}$, T. Dalkilic ${ }^{4}$, E. Kundakci ${ }^{4}$, Y. Aydin $^{4}$ \\ ${ }^{l}$ Neurosurgery, Sisli Education and Research Hospital, ${ }^{2}$ Department of Pediatric Infectious Diseases, Sisli \\ Etfal Training and Research Hospital, ${ }^{3}$ Neurosurgery, Baskent University Istanbul Hospital, ${ }^{4}$ Neurosurgery, \\ Sisli Etfal Training and Research Hospital, Istanbul, Turkey
}

Background: The rate of repeat shunt-related cerebrospinal fluid (CSF) infections and the contributing risk factors were analyzed.

Methods: Our clinic treated 58 children for initial shunt infection(ISI). Of the 58 children, 15 were diagnosed with repeat shunt infection(RSI) within 6 months of ISI treatment. For the analysis of potential risk factors for RSI, the following independent variables tested separately: a)age b)aetiology of hydrocephalus c)ISI microorganism d)initial surgical treatment of the ISI e)number of days of external CSF drainage with either an EVD or an externalized shunt, and f)presence of culture positive CSF at ISI .

Results: Cerebrospinal fluid(CSF) drainage, initiated with removal of the shunt system and insertion of external ventricular drainage(EVD) catheters in 44(75.8\%) children and with externalization of the existing ventricular catheters in $14(24.2 \%)$ of them. We detected repeat shunt infection in 15 of these children. Among these 15 RSI children, 9 were the ones who have been treated with removal of shunt and insertion of new EVD catheter while 6 were the ones who have been treated with externalization of ventricular catheters. There is a statistically significant increased number of RSI in cases which have been treated with externalization of ventricular catheter instead of removal of shunt and insertion of new EVD catheter $(\mathrm{p}=0.032,<0.05)$. None of the other variables reached statistical significance as a risk factor in our study.

Conclusion: To reduce the risk of repeat shunt infections, total removal of shunt and insertion of new EVD catheter should be preferred instead of externalization of infected ventricular catheter. 